\title{
Single perineal incision for artificial urinary sphincter: analysis of technique, outcomes, and experience
}

\author{
Nahid Punjani ${ }^{1,2}$, Ernest Chan ${ }^{2}$, Garson Chan ${ }^{2}$, Haider Abed $^{2}$, Jeffrey Campbell ${ }^{2}$, Gerald Brock $^{2}$ \\ ${ }^{1}$ Center for Reproductive Medicine and Surgery, Weill Cornell Medicine, New York, NY, USA; ${ }^{2}$ Division of Urology, Department of Surgery, \\ Schulich School of Medicine \& Dentistry, Western University, London, ON, USA \\ Contributions: (I) Conception and design: N Punjani, E Chan, G Chan, G Brock; (II) Administrative support: N Punjani, E Chan, G Brock; (III) \\ Provision of study materials or patients: G Brock; (IV) Collection and assembly of data: N Punjani, E Chan, H Abed, G Chan; (V) Data analysis and \\ interpretation: N Punjani, E Chan, G Chan, H Abed, G Brock; (VI) Manuscript writing: All authors; (VII) Final approval of manuscript: All authors. \\ Correspondence to: Gerald Brock, MD, FRCSC. Professor of Surgery, Western University, Room B4-663, St Joseph's Health Care, 268 Grosvenor \\ Street London ON N6A 4V2, USA. Email: gebrock88@sympatico.ca.
}

\begin{abstract}
Background: To describe a large series of male patients who underwent a minimally invasive single perineal incision artificial urinary sphincter (AUS) placement in patients with stress urinary incontinence.

Methods: A retrospective cohort study was performed with data collected from men undergoing AUS placement by a single high-volume surgeon over a 12-year period (2005 to 2017). Demographic and outcomes data related to AUS placement were recorded from electronic medical records, which included subjective histories and questionnaires. Institutional ethics approval was received.

Results: A total of 145 AUS were placed over the study period. Of these, 84 were performed through a single perineal incision for both device and reservoir placement. Almost all ( $\mathrm{n}=81,96 \%)$ reported preoperative incontinence of more than 3 pads per day. Postoperatively, $75 \%$ were satisfied with their continence, with 21 (25\%) complaining of recurrent incontinence. A total of 5 (6\%) patients developed a post-operative infection, 10 (12\%) had device erosion and 11 (13\%) had device malfunction, but only 3 (4\%) had reservoir dysfunction. A total of 24 (29\%) patients required revision of their device at median of 20 months (IQR, 6-32.5 months).

Conclusions: Single perineal incision is a feasible, safe, and potentially superior approach for AUS placement and warrants consideration as an accepted approach due to its more rapid surgical times, lower morbidity related to a single incision with minimal fascial defect, and favorable complication rates.
\end{abstract}

Keywords: Artificial urinary sphincter (AUS); post-prostatectomy incontinence (PPI); single incision

Submitted Feb 03, 2020. Accepted for publication Jul 16, 2020.

doi: 10.21037/tau-20-508

View this article at: http://dx.doi.org/10.21037/tau-20-508

\section{Introduction}

\section{Background}

Severe stress urinary incontinence (SUI) is one of the most distressing complications following radical prostatectomy with significant negative effects on quality of life $(1,2)$. Implantation of an artificial urinary sphincter (AUS) is the gold standard treatment for post-prostatectomy incontinence (PPI), with continence rates (as defined by a continence status of $0-1$ pads) of $59-90 \%$ and patient- reported satisfaction rates of $87-90 \%$ in long-term followup (3). Since its introduction, use of AUS has also expanded to the management of SUI caused by transurethral procedures, radiation therapy, intrinsic sphincter deficiency, neurogenic bladder, congenital disorders, and orthotopic neobladder urinary diversion $(4,5)$.

The standard device, the AMS 800 AUS, is traditionally implanted using a combined perineal and abdominal approach, which requires two incisions: a perineal incision for placement of the cuff, and a suprapubic incision for 
placement of a pressure regulating balloon (PRB) and pump (6). Advances in techniques and technology have led to the development of various other approaches, which include the transscrotal approach (single incision), abdominal approach (especially in females), combined abdominal and transvaginal approach, laparoscopic approach, and even robot-assisted approach $(7,8)$. Many of these techniques are still not widely practiced nor adopted, possibly due to higher learning curve, expensive of new equipment, or poor exposure.

In this study, we propose a novel and accessible single perineal incision technique that may be less morbid and has been used at our center for many years as only a few small series have been published on this technique (9).

\section{Objectives}

To describe the technique of implantation of an AUS through a minimally invasive single perineal incision approach. To report the postoperative complications and functional outcomes of this approach performed by a highvolume surgeon at a tertiary care centre.

We present the following article in accordance with the STROBE reporting checklist (http://dx.doi.org/10.21037/ tau-20-508).

\section{Methods}

\section{Study design}

A retrospective cohort study was performed for patients with data reviewed over a 12-year period (2005 to 2017) from a single high-volume surgeon at a tertiary academic center. All patients undergoing placement of AUS with available data were included. All implanted components were produced by American Medical Systems (AMS; Minnetonka, MN). The procedures in this study were in accordance with the Declaration of Helsinki (as revised in 2013) and approved by the Western University Research Ethics Board (REB\#110712). Because of the retrospective nature of the research, the requirement for informed consent was waived.

\section{Variables}

Demographic and outcomes data related to AUS placement were recorded from electronic medical records. Demographic data included age (in years) and body mass index $\left(\mathrm{kg} / \mathrm{m}^{2}\right)$ at the time of surgery as well as the use of alcohol ( $<2$ vs. $\geq 2$ alcoholic beverages per day) and smoking status (current or quit $v s$. never). Relevant past medical history of diabetes, cardiovascular disease, and other Charlson Comorbidity Index characteristics were documented. Additionally, previous radical prostatectomy, previous pelvic radiation, prior AUS, and previous nonAUS procedure for continence were recorded. Preoperative duration of incontinence $(\geq 1 v s .<1$ year), and pad usage ( $<3 v s$. $\geq 3$ pads per day) was recorded both pre- and post-operatively. Procedural parameters included operative time, length of stay, complications within 30 days were recorded. Postoperative complications including revision surgery for infection, erosion, and device malfunction were recorded. Time to need for revision was noted (in months).

\section{Statistical methods}

Study size was based on the number of patients with complete data for analysis identified through retrospective review. Descriptive statistics were used to describe demographic variables, assessments of urinary function, and post-operative complications. Missing data was excluded from analysis. All data was analyzed using STATA version 14.1 (StataCorp LLC, College Station, TX).

\section{Operative technique}

\section{Indications for surgery}

AUS is offered to males at our center for significant stress urinary incontinence, either due to significant leakage or patient preference after a thorough discussion about the risks and complications of a prosthetic device. Patients are not offered the device if they lack dexterity, cognition, or are poor surgical candidates.

\section{Pre-operative preparation}

Patients are optimized from a medical and anesthetic perspective including diabetic control and management. All patients were consented for insertion of an AMS AUS device.

\section{Patient preparation}

Patients receive peri-procedural intravenous antibiotics. Patients are placed in the lithotomy position; the perineal hair is trimmed with clippers and a pre-operative 5-minute scrub is completed. The perineum is then prepped with alcohol-based chlorohexidine solution and the rectum is 


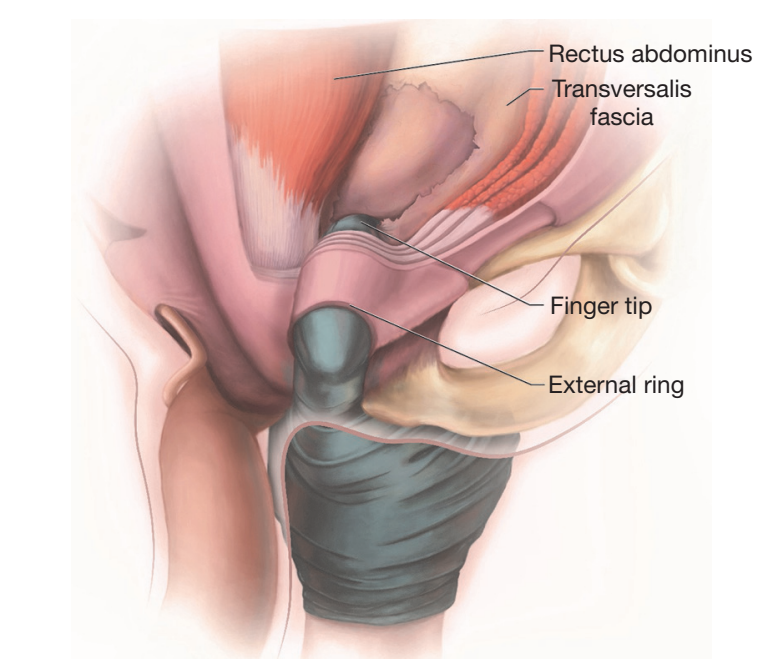

Figure 1 Depiction of access using surgeon's finger into the retropubic space for reservoir placement behind the transversalis fascia.

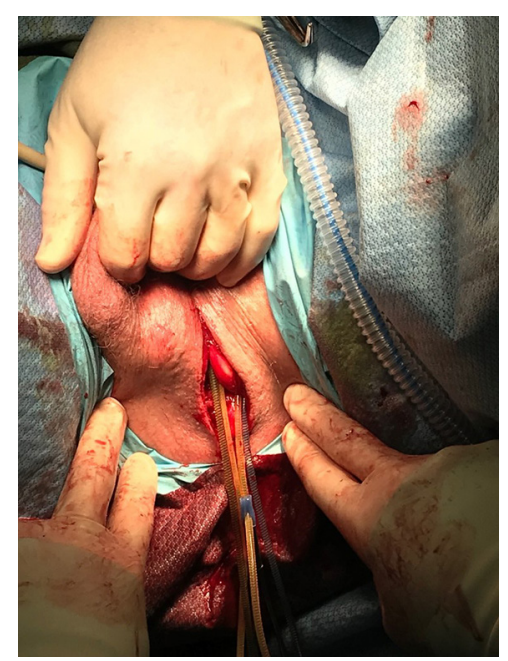

Figure 2 Illustration highlighting pump and pressure regulating balloon tubing from a single perineal incision $(3-5 \mathrm{~cm})$.

covered using sterile drapes to prevent contamination. Proviodine solution is injected per urethra with a $20 \mathrm{~mL}$ syringe, a Foley catheter is inserted under sterile conditions, and the bladder is emptied into a basin, avoiding contamination of the surgical field.

\section{Placement of the pressure regulating balloon}

A 3 to $5 \mathrm{~cm}$ vertical incision is made in the perineum and dissection carried down to the spongiosis muscles, which are sharply divided. The urethra is isolated and cuff size is measured. The retropubic space is developed by locating the external inguinal ring, followed by blunt dissection and gentle entrance through the transversalis fascia as seen in Figure 1. A small retractor (i.e., 5/8" Deaver) may be used to apply traction superiorly within the external inguinal ring to create tension on the transveralis fascia and facilitate blunt dissection.

Care should be taken not to develop a space that is larger than necessary for the inflated balloon. The entrance to the retropubic space should be narrow enough to prevent herniation of the pressure regulating balloon (PRB), which requires minimization of excessive dissection by the surgeon and trainees. Once the positioned and filled with $24 \mathrm{~mL}$ of saline, the PRB is assessed for risk of herniation. The cuff is placed around the urethra. Figure 2 illustrates all tubing emanating from the single 3 to $5 \mathrm{~cm}$ perineal incision prior to pump placement.

\section{Placement of the valve pump}

A subdartos pouch is developed in an accessible location on the anterior aspect of the scrotum by invaginating the scrotum through the perineal incision using a finger placed externally on the scrotum. Dartos fascia is bluntly swept away over the desired position of the valve pump (VP). The $\mathrm{VP}$ is deployed in this subdartos pouch and secured with a 3-0 PDS suture.

\section{Flexible cystoscopy and closure}

The foley catheter is removed and flexible cystoscopy is performed to assure complete occlusion with device activation; following which the device is deactivated. The perineal incision is closed in multiple layers using absorbable sutures. No drains are placed. A compressive turban-type bandage with cling gauze is applied to minimize postoperative hematoma and to fix the VP in position. The final device placement is shown in Figure 3.

\section{Post-operative care and follow-up}

Post-operatively, patients are admitted overnight for a 24-hour period of intravenous antibiotics. No Foley catheter is required postoperatively and the AUS is left deactivated. Patients are typically discharged home on postoperative day one following removal of the compressive dressing, assessment of the wound, and monitoring of postvoid residual bladder volumes. Prescription is provided for a two-week course of oral fluoroquinolone antibiotic and analgesics. A standard antibiotic duration was determined 


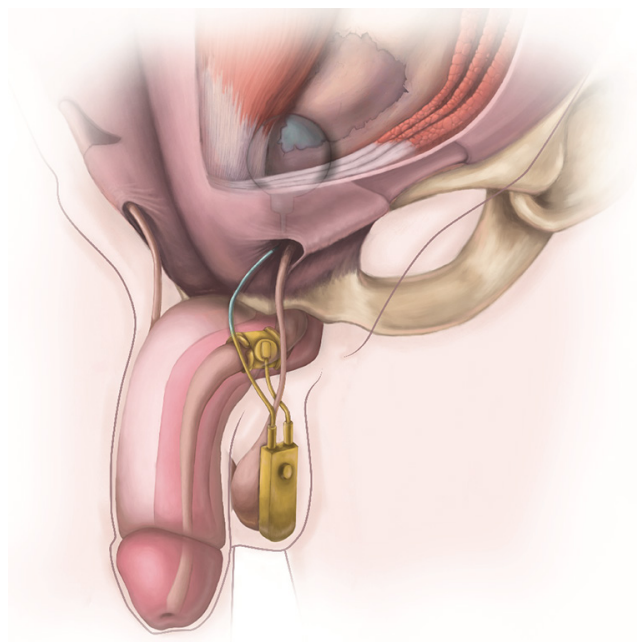

Figure 3 Illustration demonstrating the location of the reservoir behind the pubic bone and transversalis fascia, as well as the device cuff around the urethra and pump in the dependent portion of the scrotum.

Table 1 Patient demographics of men undergoing single perineal incision AUS ( $\mathrm{n}=84)$

\begin{tabular}{lc}
\hline Parameter & Values \\
\hline Age, years (median, IQR) & $71(64$ to 74$)$ \\
BMI, kg/m² (median, IQR) & $27(25$ to 30$)$ \\
Smoking (current or previous), $\mathrm{n}(\%)$ & $33(39.3)$ \\
Daily alcohol use, $\mathrm{n}(\%)$ & $6(7.1)$ \\
Diabetic, $\mathrm{n}(\%)$ & $13(15.5)$ \\
History of myocardial infarction, $\mathrm{n}(\%)$ & $25(29.8)$ \\
Hypertension, $\mathrm{n}(\%)$ & $45(53.6)$ \\
Radical prostatectomy, $\mathrm{n}(\%)$ & $79(94.0)$ \\
Pelvic radiation, $\mathrm{n}(\%)$ & $35(41.7)$ \\
\hline
\end{tabular}

IQR, interquartile range.

Table 2 Urinary function parameters of men undergoing single perineal incision AUS $(n=84)$

\begin{tabular}{lc}
\hline Parameter & Number (\%) \\
\hline Pre-op duration of incontinence (<1 year) & $82(97.6)$ \\
Pre-op incontinence (>3 pads per day) & $81(96.4)$ \\
Prior non-AUS surgery for incontinence & $7(8.3)$ \\
Prior AUS & $15(17.9)$ \\
Post-op continence (0 or precautionary pad) & $63(75.0)$ \\
\hline
\end{tabular}

based on the increased risk of infection in our cohort due to prevalence of previous pelvic radiation, diabetes, and/or previous continence surgeries. Follow-up occurs six weeks post-operatively for wound assessment, sphincter activation, and teaching.

\section{Results}

A total of 145 patients were identified through retrospective chart review and examined for eligibility. All patients were confirmed eligible based on complete data available for analysis and included in the study. Eighty-four (57.9\%) were placed using a single perineal incision. The majority of the remainder were single incision transscrotal procedures, which has been previously described (7). Most AUS were placed between 2011 to 2017 ( $n=83$; mean 13.8 AUS per year) and the most placed in a single year was 19 (in 2011).

Median age of patients undergoing single perineal incision AUS placement was 71 years and median Charlson comorbidity index was 6. Median follow-up duration was 21 months. Patient demographic data and past medical history are shown in Table 1 .

Of the 84 patients who underwent single perineal incision AUS placement, 81 patients $(96 \%)$ reported preoperative SUI requiring more than 3 pads per day and 82 (97.6\%) had SUI for less than 1 year (Table 2). Moreover, 15 (17.9\%) had previous AUS procedure, and 7 (8.3\%) had a previous non-AUS treatment of their SUI, such as urethral bulking agents, male slings, etc. The etiology of SUI in our sample was primarily radical prostatectomy $(n=79 ; 94 \%)$. Importantly, almost half of our patients had previous pelvic radiation $(\mathrm{n}=35,41.7 \%)$.

Post-operative parameters are listed in Table 3. The median length of stay in hospital was 1 day. Long-term complications over the entire study period included a total of $5(6 \%)$ patients with a post-operative infection, 10 (12\%) patients had device erosion, 11 (13\%) had a device malfunction, and $3(4 \%)$ with PRB dysfunction. A total of $24(29 \%)$ patients required revision of their device at median of 20 months (IQR, 6-32.5 months). There were no reported complications from urethral atrophy during follow up. Patients with previous pelvic radiation had increased risk of short-term and long-term post-operative complications and required reoperation sooner (Table 3).

\section{Discussion}

AUS placement remains the gold standard for post- 
Table 3 Post-operative parameters of men undergoing single perineal incision AUS

\begin{tabular}{|c|c|c|}
\hline Parameter & $\begin{array}{l}\text { All patients } \\
\quad(n=84)\end{array}$ & $\begin{array}{l}\text { Radiation } \\
(\mathrm{n}=35)\end{array}$ \\
\hline Operative time, min (median, range) & 51 (44 to 59$)$ & 50 (42 to 58$)$ \\
\hline Length of stay, days (median, range) & 1 (1 to 3$)$ & 1 (1 to 2$)$ \\
\hline Follow-up, months (median, IQR) & 21 (12 to 44$)$ & 21 (7 to 43$)$ \\
\hline Infection*, n (\%) & $5(6.0)$ & $2(5.7)$ \\
\hline Erosion*, n (\%) & $10(11.9)$ & $5(14.3)$ \\
\hline Malfunction*, n (\%) & $11(13.1)$ & $5(14.3)$ \\
\hline Need for revision*, n (\%) & $24(28.6)$ & $13(37.1)$ \\
\hline $\begin{array}{l}\text { Time to revision, in months (median, } \\
\text { IQR) }\end{array}$ & 20 (6 to 33$)$ & 14 (6 to 29$)$ \\
\hline Complication within 30 days, $\mathrm{n}(\%)$ & $6(7.1)$ & 5 (14.3) \\
\hline Retention (catheterization) & $4(4.8)$ & $4(11.4)$ \\
\hline Leg pain & $1(1.2)$ & $0(0.0)$ \\
\hline Herniated reservoir & $1(1.2)$ & $1(2.9)$ \\
\hline
\end{tabular}

*, over 12-year period.

prostatectomy incontinence patients (1). Traditional studies have illustrated the use of a counter-incision for PRB placement, and only a few small series have described a single perineal incision approach to AUS placement (9). This study represents the largest series to our knowledge utilizing this novel approach and highlights its feasibility and practicality as an alternative method of AUS placement, with comparable complication rates, and provides excellent cosmetic results with no visible groin incisions.

The traditional two-incision technique has changed minimally since the development of the AMS AUS for SUI, with the basic design unchanged since 1983 (10). Disadvantages of this technique include patient discomfort and inferior cosmesis from the inguinal counter-incision, and longer operating times needed to open and close a second incision. As a consequence, other devices have been trialed but have controversial results; including the Flow Secure device, ZSI 375, and Periurethral Constrictor (11). In addition, a second incision may be difficult to perform in certain cases, especially in those with history of inguinal hernia or mesh placement. The counter-incision itself can potentially cause a hernia, whereas single incision techniques (i.e., transscrotally) have shown no hernia occurrences in modern series, even in high-risk patients (12). Foreign body such as hernia mesh, moreover, can make the dissection more complicated with possible increase in risk of device infection. Other techniques have been advocated but these often require specialized equipment or difficult learning curves, such as a robot-assisted approach (13).

The only relative contraindications to the single perineal incision approach occur in cases where the surgeon believes or anticipates difficulty placing the PRB, owing to expected fibrosis, previous hernia repairs, or situations where blind placement may represent a heightened degree of risk (e.g., following a renal transplant or femorofemoral bypass). In some cases, additional tools may be necessary, such as gentle perforation of the scarred transversalis fascia using a sharp Metzenbaum scissors, which has been previously described. The majority of single perineal incision PRB placements at our center, however, are completed with blunt finger dissection in order to minimize the risk of unintended injury to surrounding structures (14).

In cases where attempted blind PRB placement is difficult or the anatomy is distorted, conversion to an open placement is easily performed using a small inguinal counter-incision as per routine. This has been rarely required in our experience, despite high rates of previous radical prostatectomy (94\%) or continence surgery (26.2\%). Furthermore, a large subset of our sample had previous pelvic radiation (41.7\%), which was noted to be higher than other published studies (15). Previous radiation typically makes dissection more challenging, resulting in a greater risk of short-term and long-term complications (Table 3). The high proportion of patients with prior radiation or surgery in this study highlights how this novel technique is feasible even in patients with fibrotic and scarred tissue (16).

In the setting of previous inguinal hernia repair with mesh, the contralateral side is selected for PRB placement. For those who have had bilateral inguinal hernia repair with mesh, a blind blunt dissection is usually still performed and often there remains an adequate space for placement of the PRB at the location where the spermatic cord exits the inguinal canal. If this space is fibrotic and stenotic, we would choose to create a small inguinal counter-incision and place the PRB under direct vision.

Patient continence rates were similar to some large series reported in the literature $(17,18)$. This illustrates the feasibility of this technique as a reasonable option for patients from a functional perspective. Over a 12-year period of time, the complication rates in our series were in keeping with that published in the literature with revision rates published in a large series at 34\% (19). Our rates of infection $(6 \%)$ were just slightly higher than those 
reported in multiple series ranging from $1.4-5.5 \%$, but this is likely a reflection of a higher proportion of medically complex patients (i.e., prior radiation, pelvic surgery, failed continence surgeries) referred to our institution (15,20-22). The infection rate in those who underwent a single transscrotal incision was similar $(\mathrm{n}=3,5 \%)$ to those who had a single perineal incision. Published rates of erosion (4$25 \%)$ and malfunction (6-33\%) were similar to our study (15,20-22). Although no formal questionnaire was completed for post-operative patient satisfaction with regards to non-leakage issues, many patients anecdotally reported increased satisfaction with regards to cosmetic outcomes. Many patients were often referred to our center with the desire to have their procedure completed in this fashion due to the potential cosmetic results.

Our median skin-to-skin operating time was 50.5 minutes, understanding that many components of the case are done by residents in a teaching environment. While operative times are variable in the literature, a single-incision approach has been shown to decrease this duration (7). Many previous studies have shown that longer operative times are associated with increased risk of infection (23). Any technique to mitigate this, therefore, is reasonable. Additionally, the learning curve for trainees is reasonable and short, especially compared to other novel techniques such as a robot-assisted approach. Chief residents and fellows (1 year) at our institution become familiar with this technique by the time they complete their training, as the placement of the PRB is identical to that of a blind threepiece inflatable penile prosthesis reservoir placement.

Limitations of our study include the retrospective design. A comparison between single perineal incision and traditional two-incision approach was not performed as the latter represented a more complex population in which a counter incision was necessary (i.e., retrieval of previous $\mathrm{PRB}$ migrated into the retroperitoneum). Moreover, our study relied on self-reported patient outcomes such as patient satisfaction and number of pads used, as opposed to validated quality of life questionnaires or pad weight assessments. Future prospective evaluations of this technique should include validated and objective measures of patient outcomes and quality of life.

Our study had limited follow-up, as many of these patients are referred to our academic tertiary care center and long-term follow-up is carried out by their local urologist if there are no significant postoperative complications (urinary retention, difficulty activating device, infection/ erosion). Otherwise, patients were followed at our centre in regular intervals. Although some patients may not have followed-up at our centre, the stated complication rates are similar to those reported in the literature. Furthermore, our reported complication rates are believed to be realistic and accurate, as we are the main referral center in the region for reconstructive urology. Very few nearby centers are experienced with the management of AUS complications, and in the context of the publicly funded healthcare system in Canada, we see most of the complications that occur from our implanted devices.

\section{Conclusions}

The AUS is a proven treatment for post-prostatectomy incontinence that arguably should be placed through a single perineal incision. This technique allows for excellent exposure and permits implantation of all three device components. The current study represents the largest data series to date on the single perineal incision technique performed. Our experience and outcomes illustrate the safety and satisfaction of patients undergoing this procedure. This technique has similar outcomes compared to other techniques, with a low learning curve. Both patient and surgeon satisfaction are high, and this approach offers reduced surgical time and a compelling alternative to the traditional AUS placement. Future series with longer follow-up and compared outcome analysis will allow for better understanding and patient education regarding available approaches for AUS placement.

\section{Acknowledgments}

The authors would like to acknowledge Dani Sayeau for medical illustration.

Funding: NP is supported in part by the Frederick J. and Theresa Dow Wallace Fund of the New York Community Trust.

\section{Footnote}

Reporting Checklist: The authors have completed the STROBE reporting checklist. Available at http://dx.doi. org/10.21037/tau-20-508

Data Sharing Statement: Available at http://dx.doi. org/10.21037/tau-20-508

Conflicts of Interest: All authors have completed the ICMJE 
uniform disclosure form (available at http://dx.doi. org/10.21037/tau-20-508). GB serves as an unpaid editorial board member of Translational Lung Cancer Research from Mar 2018 to Feb 2020. GB reports other from Boston Scientific, Eli Lilly, Acerus Pharma, Pfizer, Paladin, Merck, Upjohn, outside the submitted work. The authors have no other conflicts of interest to declare.

Ethical Statement: The authors are accountable for all aspects of the work in ensuring that questions related to the accuracy or integrity of any part of the work are appropriately investigated and resolved. The procedures in this study were in accordance with the Declaration of Helsinki (as revised in 2013) and approved by the Western University Research Ethics Board (REB\#110712). Because of the retrospective nature of the research, the requirement for informed consent was waived.

Open Access Statement: This is an Open Access article distributed in accordance with the Creative Commons Attribution-NonCommercial-NoDerivs 4.0 International License (CC BY-NC-ND 4.0), which permits the noncommercial replication and distribution of the article with the strict proviso that no changes or edits are made and the original work is properly cited (including links to both the formal publication through the relevant DOI and the license). See: https://creativecommons.org/licenses/by-nc$\mathrm{nd} / 4.0 /$.

\section{References}

1. Peyromaure M, Ravery V, Boccon-Gibod L. The management of stress urinary incontinence after radical prostatectomy. BJU Int 2002;90:155-61.

2. Caremel R, Corcos J. Incontinence after radical prostatectomy: Anything new in its management? Can Urol Assoc J 2014;8:202-12.

3. Herschorn $S$. The artificial urinary sphincter is the treatment of choice for post-radical prostatectomy incontinence. Can Urol Assoc J 2008;2:536-9.

4. Kowalik CG, Dmochowski RR, De EJB. Surgery for female SUI: The ICI algorithm. Neurourol Urodyn 2019;38 Suppl 4:S21-7.

5. Ludwikowski BM, Bieda JC, Lingnau A, et al. Surgical Management of Neurogenic Sphincter Incompetence in Children. Front Pediatr 2019;7:97.

6. Chouhan JD, Terlecki RP. A User's Guide for Surgery Involving the Artificial Urinary Sphincter. Sex Med Rev
2019;7:167-77.

7. Wilson S, Delk J 2nd, Henry GD, et al. New surgical technique for sphincter urinary control system using upper transverse scrotal incision. J Urol 2003;169:261-4.

8. Peyronnet B, O'Connor E, Khavari R, et al. AMS-800 Artificial urinary sphincter in female patients with stress urinary incontinence: A systematic review. Neurourol Urodyn 2019;38 Suppl 4:S28-41.

9. Benson CR, Ayoub HI, Westney OL. Single perineal incision placement of artificial urinary sphincter with cadaveric correlation of sub-dartos pump placement. Int Braz J Urol 2018;44:355-61.

10. Montague DK. Artificial urinary sphincter: longterm results and patient satisfaction. Adv Urol 2012;2012:835290.

11. Vakalopoulos I, Kampantais S, Laskaridis L, et al. New artificial urinary sphincter devices in the treatment of male iatrogenic incontinence. Adv Urol 2012;2012:439372.

12. Sotelo TM, Westney OL. Outcomes related to placing an artificial urinary sphincter using a single-incision, transverse-scrotal technique in high-risk patients. BJU Int 2008;101:1124-7.

13. Herve F, Lumen N, Goessaert AS, et al. Persistent urinary incontinence after a robot-assisted artificial urinary sphincter procedure: lessons learnt from two cases. BMJ Case Rep 2016;2016:bcr2016216971.

14. Al-Shaiji TF. Dual implantation of artificial urinary sphincter and inflatable penile prostheses for concurrent male urinary incontinence and erectile dysfunction. Adv Urol 2011;2011:178312.

15. Lai HH, Hsu EI, Teh BS, et al. 13 years of experience with artificial urinary sphincter implantation at Baylor College of Medicine. J Urol 2007;177:1021-5.

16. Srivastava A, Joice GA, Patel HD, et al. Impact of Adjuvant Radiation on Artificial Urinary Sphincter Durability in Postprostatectomy Patients. Urology 2018;114:212-7.

17. Tutolo M, Cornu JN, Bauer RM, et al. Efficacy and safety of artificial urinary sphincter (AUS): Results of a large multi-institutional cohort of patients with mid-term follow-up. Neurourol Urodyn 2019;38:710-8.

18. Yafi FA, Powers MK, Zurawin J, et al. Contemporary Review of Artificial Urinary Sphincters for Male Stress Urinary Incontinence. Sex Med Rev 2016;4:157-66.

19. Radomski SB, Ruzhynsky V, Wallis CJD, et al. Complications and Interventions in Patients with an Artificial Urinary Sphincter: Long-Term Results. J Urol 
2018;200:1093-8.

20. Gousse AE, Madjar S, Lambert MM, et al. Artificial urinary sphincter for post-radical prostatectomy urinary incontinence: long-term subjective results. J Urol 2001;166:1755-8.

21. Leon P, Chartier-Kastler E, Roupret M, et al. Longterm functional outcomes after artificial urinary sphincter implantation in men with stress urinary incontinence.

Cite this article as: Punjani N, Chan E, Chan G, Abed H, Campbell J, Brock G. Single perineal incision for artificial urinary sphincter: analysis of technique, outcomes, and experience. Transl Androl Urol 2020;9(5):1912-1919. doi:10.21037/ tau-20-508
BJU Int 2015;115:951-7.

22. Elliott DS, Barrett DM. Mayo Clinic long-term analysis of the functional durability of the AMS 800 artificial urinary sphincter: a review of 323 cases. J Urol 1998;159:1206-8.

23. Brant WO, Martins FE. Artificial urinary sphincter. Transl Androl Urol 2017;6:682-94. 